\title{
Les femmes, les hommes, le féminisme
}

Jean Martin

gramme pour un essai artistico-philosophico-littéraire qui est un peu un cri du cœur.

Il le fait par une quarantaine de collages, un passe-temps auquel il s'adonne depuis trente ans (avec jubilation, dit-il), en rapport avec la nature - et la culture - des femmes et des hommes, et leurs relations. Rassemblement et arrangement stimulant, très varié, d'illustrations prises ici et là, pour l'essentiel au cours du XIX ${ }^{\mathrm{e}}$ (seconde moitié) et du XX ${ }^{\mathrm{e}}$ siècles. Avec un texte qui lie le tout, en chapitres où son expérience de médecin parlant avec des femmes et les traitant joue un rôle d'importance. Souvent drôle, humour au premier et au second degrés. Quelques titres: «Chassés du Paradis», «Et la honte fut», «De quelques nuances d'ordre physique... et psychique», «Le mys-

\section{«Pourquoi cette manie, cette rage de confondre «différent〉 avec 〈inférieur〉 qui sent si fort la revendication politique?» (Alain Noyer)}

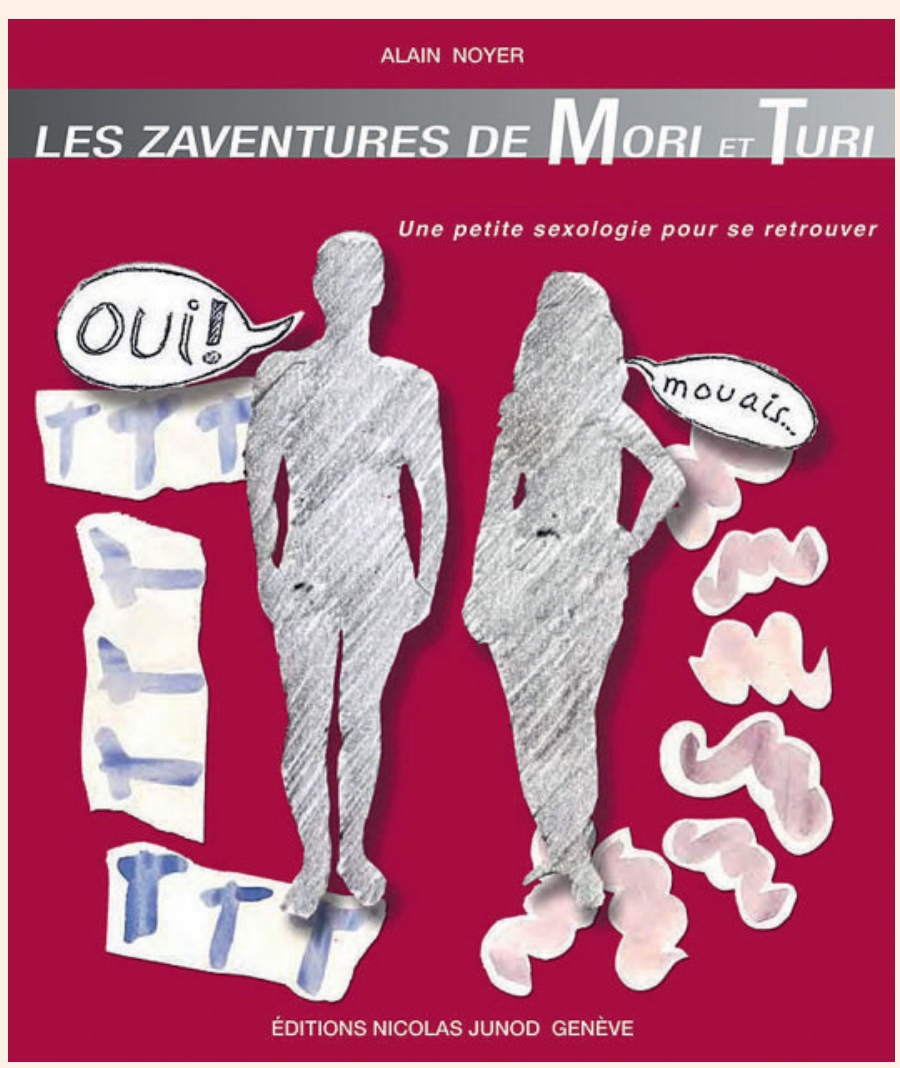

tère féminin», «Une pub sexiste?», «Mariages en tous genres», «La si belle égalité», parmi d'autres.

Des choses avec lesquelles nous sommes tous d'accord: quand il déplore la «chosification» de la femme - parmi d'autres chosifications dont nous sommes témoins; lorsqu'il stigmatise la publicité sexiste, à l'évidence un thème majeur et déplorable des paysages médiatiques. Des considérations sur la

Alain Noyer

Les Zaventures de Mori et Turi.

Une petite sexologie

pour se retrouver.

Genève: Editions Nicolas Junod;

2010. 128 pages. $32 \mathrm{CHF}$. 
Un collage de Alain Noyer sur les femmes et les hommes, et leurs relations.

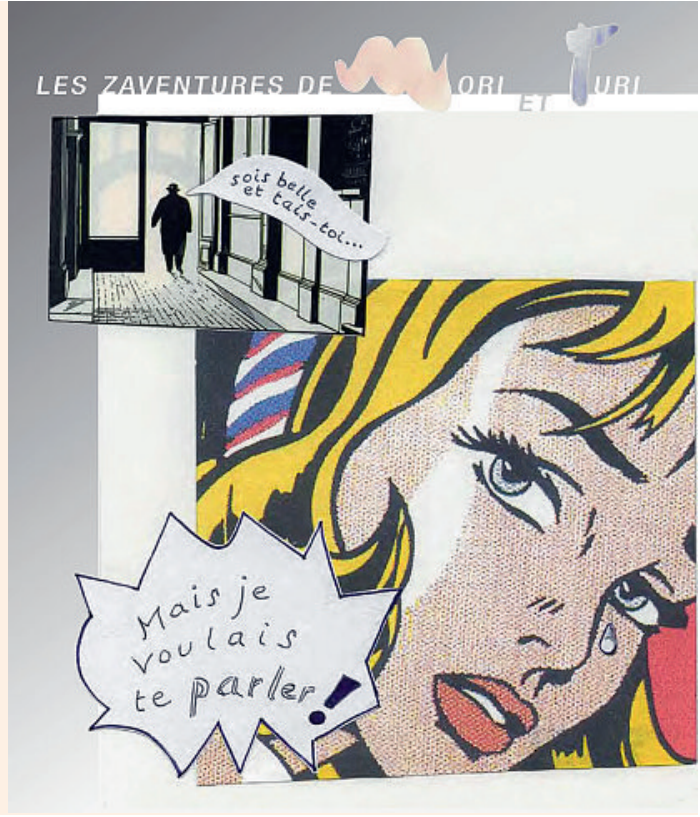

manière dont la différence de genre peut s'exprimer dans la création artistique. Plus téméraire: «Aborder les différences d'ordre psychique est plus délicat. Beaucoup plus délicat; risque non négligeable de morsure par une chienne de garde. Risque de procès....»

«L'homme et la femme sont égaux en droits, bien sûr, mais ils sont différents de corps et d'idées.» D'accord. «Bref, à vouloir coûte que coûte donner à la femme des caractéristiques qu'elle n'a généralement pas, et enlever à l'homme des particularités qu'il a en général - et vice versa, on s'éloigne d'une compréhension intelligente de ce qui fait l'originalité et la richesse de chaque sexe.» Oui, on se rallie... toutefois certains demanderont à discuter plus avant du «en général»; il n'est pas exclu que des exceptions à la règle générale interpellent. «Pourquoi cette manie, cette rage de confondre «différent s avec «inférieur〉 qui sent si fort la revendication politique?». Well, well... Je ne me défais pas d'un peu de malaise quant aux possibles implications de cette question: il faut tout de même se méfier des applications (rien que dans le passé récent, dans différents régimes) de formules comme «different but equal» ou «separate but equal». Etant entendu qu'il est clair qu'Alain Noyer ne souhaite pas un apartheid des genres!

L'auteur n'est pas un soutien inconditionnel des Offices de l'égalité entre femmes et hommes: «Les Bureaux pour l'Egalité sont frappés d'un mal étrange. Leurs parois s'effritent, les portes ferment mal et les tiroirs coincent... L'égalité n'a plus la vertu qu'on lui croyait.» Que dire? Sans doute y a-t-il des démarches maxima- listes qui vont/allaient au-delà du bon sens - tel que pour ma part je le vois. Mais je ne voudrais pas que ces Bureaux disparaissent aujourd'hui, il y a encore à faire.

Même réserve ironique d'Alain Noyer à propos du langage épicène (de la rage épicénique). Tout en relevant l'importance de la dimension culturelle, je peux le suivre dans une certaine mesure (je n'arrive pas à m'enthousiasmer pour la systématique des «PatientInnen», «KundInnen» ou «ÄrztInnen» de nos compatriotes alémaniques - logique mais bien lourd). Mais je suis critique de notre grand voisin hexagonal dans sa résistance à aménager le langage; en France, le respect d'une tradition terminologique paraît parfois dangereusement proche d'un machisme sommaire. On a appelé Marguerite Yourcenar, première femme à entrer à l'Académie française, Madame l'Académicien. Une femme à la tête d'une instance officielle était Madame le Président, c'est l'épouse d'un président qu'on appelle/appelait Madame la Présidente. Tout de même!

Voilà pour quelques réserves, en toute collégialité. $\mathrm{Au}$ reste, «Les Zaventures de Mori et Turi» est un objet, agréable à voir et à lire, bien écrit, aimable même quand il critique. J'y sens une tendresse de l'auteur (en tout bien tout honneur) pour les êtres humains

qu'il a côtoyés dans sa vie - des femmes dans son activité professionnelle, des hommes aussi ailleurs. Et il est bon que certains d'entre nous, arrivés au terme de la vie dite «active», transmettent leur expérience; leur vision de l'existence, de la société et de celles et ceux qui la constituent. Alain Noyer le fait sous une forme originale et concise.

«Je propose l'Armistice, la fin des hostilités, la paix des braves (brave: mot quelque peu ambigu, mais épicène après tout)», dit-il dans son dernier chapitre. Certain(e)s jugeront-ils/elles qu'il y a des choses à débattre sérieusement avant d'accepter l'armistice? Dans la mesure où on ne se laisse pas froisser par l'une ou l'autre phrase, il me semble que ce petit ouvrage apporte sa pierre, comme le veut son sous-titre, à l'idée de «se retrouver».

Sans rapport direct avec le sujet du livre mais parce que je suis un amoureux des belles formules, je termine avec ces dernières lignes des «Zaventures» - reprises de Marguerite Yourcenar dans les «Mémoires d'Hadrien»: «trois lignes sinueuses, étirées à l'infini, sans cesse rapprochées et divergeant sans cesse: ce qu'un homme (ou une femme) a cru être, ce qu'il a voulu être, et ce qu'il fut.» Avec ce livre, peutêtre Alain Noyer a-t-il voulu, pour ce qui le concerne, tenter ce rapprochement? 